\title{
Modelo Matemático para vaciado de Tanques
}

\author{
Mathematical Modeling for emptying tanks
}

\author{
Luis Fernando Plaza Gálvez \\ Unidad Central del Valle del Cauca, Tuluá, Valle, Colombia \\ lplaza@uceva.edu.co
}

\begin{abstract}
Resumen- En este trabajo, se presenta la modelación matemática que hay entre la altura del nivel de un tanque y el tiempo que toma en su vaciado. Para su objetivo se tendrán en cuenta 3 modelos con soporte en Mínimos Cuadrados, usando ecuaciones diferenciales ordinarias que son resueltas por separación de variables. Al final se realiza el cálculo del coeficiente de determinación.
\end{abstract}

Palabras clave- Ecuación Diferencial, Mínimos Cuadrados, Modelamiento Matemático, Tanque.

\begin{abstract}
By means of this proposal, it present the mathematical modeling, that is the height of the tank level and the time it takes emptying occurs. For your purpose will be considered 3 models with least squares support, using ordinary differential equations, that are solved by separation of variables. At the end of the calculation of the coefficient of determination is made.
\end{abstract}

Key Word - Differential Equation, Least Squares, Mathematical Modeling, Tank.

\section{INTRODUCCIÓN}

Es importante predecir el tiempo que demora en vaciarse total o parcialmente el contenido de un tanque o conocer la cantidad de volumen que se desaloja o queda a un determinado tiempo. En algunas oportunidades, se requiere conocer, el momento en el que se puede descargar una cantidad especial de líquido, o si es el caso realizar una mezcla, o interactuar con un sistema de control de nivel. El vaciado de Tanques, así como la transferencia de líquidos entre recipientes son comunes en plantas de producción de hidrocarburos, industrias procesadoras de alimentos (bebidas, lácteos, etc.). Permitiendo con lo anterior llevar a cabo diferentes procesos de planeación. La mecánica de fluidos permite estudiar este tipo de procesos, y como practica universitaria, permite abordar tópicos de Operaciones Unitarias.

Por medio de este trabajo, se permite dar una charla de las experiencias realizadas con el curso de Ecuaciones Diferenciales en la Unidad Central del Valle del Cauca, llevadas a cabo en el laboratorio de Ingeniería. Para ello se analiza inicialmente el comportamiento del proceso de
Vaciado de un Tanque, y la forma como varia la altura de líquido a través del tanque.

Al inicio se hace un análisis de lo que se ha estudiado al respecto, en lo que tiene que ver con la Ley de Torricelli, pero donde si se tiene en cuenta la geometría del recipiente, posteriormente se procede a realizar modelamiento con la regresión lineal, que a pesar de no ser mala su aproximación, esta se mejora ostensiblemente empleándola igual como herramienta estadística, pero después de haber hecho diferenciación numérica y originando las diferentes ecuaciones diferenciales, que las regresiones lineal, exponencial y potencial permitan, así como sus respectivas soluciones. Finalmente se llega a una expresión matemática de la altura del nivel del líquido en el tanque en función del tiempo, pero no se tiene en cuenta la geometría del recipiente y sus agujeros.

\section{ANTECEDENTES}

En hidrodinámica, la ley de Torricelli establece que la velocidad $v$ del flujo (salida) del agua a través de un orificio en la parte lateral o en el fondo de una tanque lleno con agua hasta una altura (o profundidad) $h$ es igual a la velocidad de un objeto (en este caso una gota de agua), que cae libremente desde una altura $\mathrm{h}$; esto es, $\sqrt{2 g h}$, donde g es la aceleración de la gravedad. Esta última expresión se origina al igualar la energía cinética $m v^{2} / 2$, con la energía potencial, $m g h$, luego de despejar $v$.

Supongamos que un tanque lleno de agua se deja vaciar por un agujero, por la acción de la gravedad. Se desea determinar la profundidad, h, del agua que queda en el tanque (ver figura 1 ) en el momento t.

Si el área transversal del agujero es $A_{0}$, y la velocidad del agua que sale del tanque es $\sqrt{2 g h}$, el volumen de agua que sale del tanque, por segundo, es $A_{0} \sqrt{2 g h}$. Así, si

$V(t)$ representa al volumen del agua en el tanque en cualquier momento $t$.

$$
\frac{d V}{d t}=-A_{0} \sqrt{2 g h}
$$




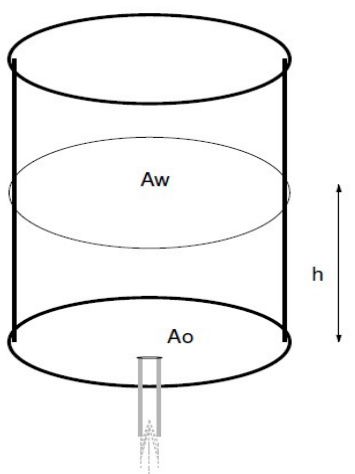

Figura 1: Diagrama Tanque cilíndrico. Ley de Torricelli

Si el tanque es tal que el volumen del agua en cualquier momento $t$ se expresa como $V(t)=A_{w} h$, donde $A_{w}$ es el área constante de la superficie superior del agua.

$$
\frac{d V}{d t}=A_{w} \frac{d h}{d t}
$$

Al reemplazar está última en (1) se llega a

$$
\frac{d h}{d t}=-\frac{A_{0}}{A_{w}} \sqrt{2 g h}
$$

Donde (3) es una ecuación diferencial por separación de variables, donde su solución es:

$$
h=f(t)=\left[\sqrt{h_{\max }}-\frac{A_{0}}{A_{w}} \sqrt{\frac{g}{2}} t\right]^{2}
$$

con hmax, como el máximo nivel de altura del agua que tiene el tanque para $\mathrm{t}=0$. Es de anotar que esta es una aplicación expuesta en textos de Ecuaciones Diferenciales como lo menciona [3].

\section{MODELO PROPUESTO}

Se desea modelar el vaciado de un tanque, cuyas dimensiones aparecen en la figura No. 1.2. El cual es llenado inicialmente de un líquido, que para nuestro caso es agua. El tanque cuenta con un orificio lateral, ubicado en el fondo, el cual regula el paso del líquido en su salida. En una fase inicial, el tanque se encuentra según se observa en las figuras No. 1.3 y 1.4 .

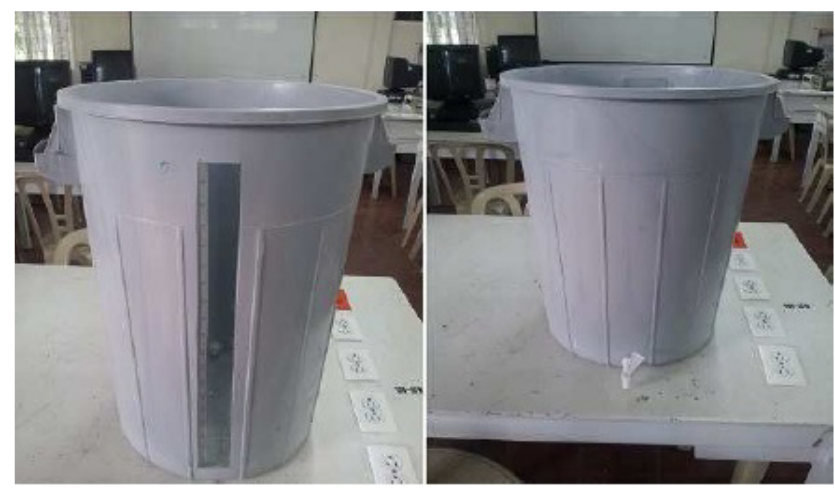

Figura 2: Tanque Vacio. Vistas frontal y trasera

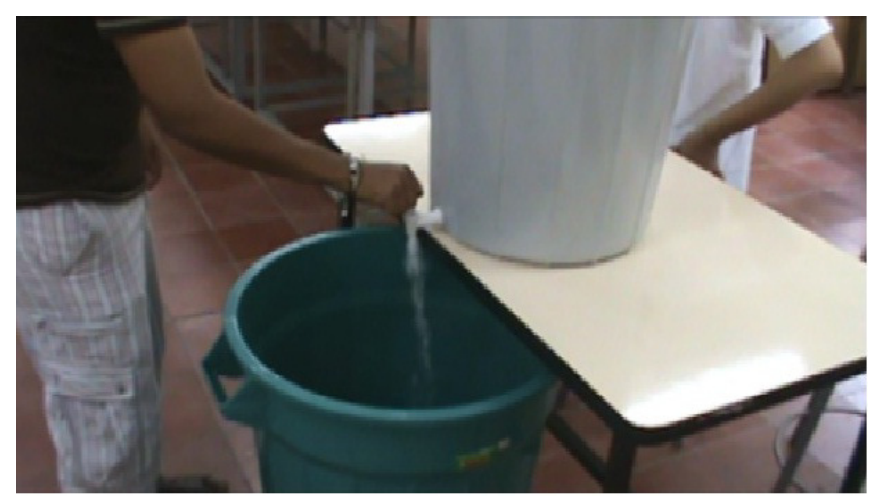

Figura 1.3: Vaciado Tanque - Fase 1

\section{TOMA DE DATOS}

En la figura No. 5, se toman los ceros de inicio de lectura, tanto en tiempo, como en altura. Es importante notar que el cero de la lectura, no coincide con el cero de la altura, de tal manera que a medida que el agua vaya descendiendo, y haga su paso por el punto cero de la referencia, sea este el instante en que se active el cronómetro, e inicie la toma de datos, los cuales quedaron consignados en la tabla No 1. Cada 30 segundos, se tomó la altura respectiva, teniendo en cuenta que ésta en realidad es tomada desde la base, y no como aparece medida, se debió restar a cada dato leído, una misma cifra que en nuestro caso fue de $45.7 \mathrm{~cm}$.

Para una mejor confiabilidad en la toma de datos, toda la práctica se filmó, de tal manera que quedará el registro histórico, y así poder confrontar datos en un momento determinado, tal como se evidencia en la figura No. 6 . 


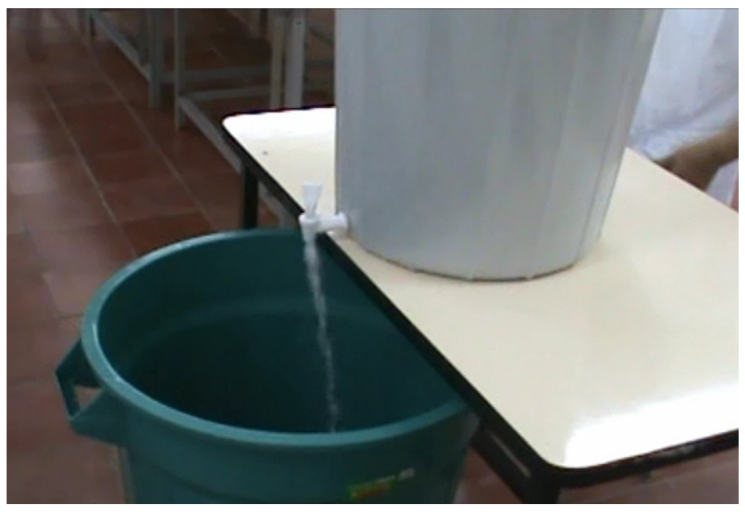

Figura 4: Vaciado Tanque - Fase 2

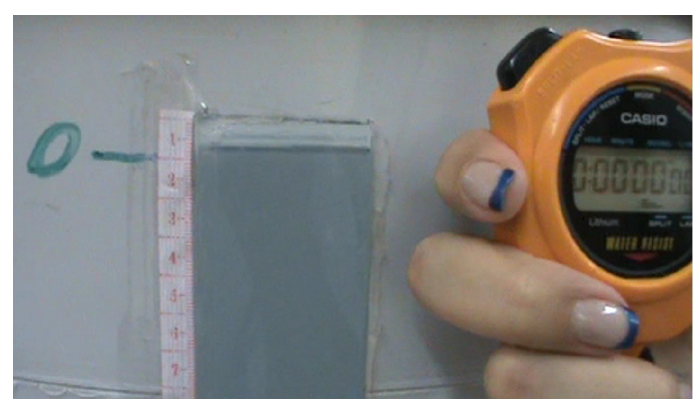

Figura 5: Toma de datos para $t=0$

\begin{tabular}{|c|c|}
\hline$t(\min )$ & $\mathrm{h}(\mathrm{cm})$ \\
\hline 0 & 45,7 \\
\hline 0,5 & 43 \\
\hline 1 & 40,4 \\
\hline 1,5 & 37,8 \\
\hline 2 & 35,2 \\
\hline 2,5 & 32,8 \\
\hline 3 & 30,3 \\
\hline 3,5 & 27,9 \\
\hline 4 & 25,6 \\
\hline 4,5 & 23,3 \\
\hline 5 & 21,1 \\
\hline 5,5 & 18,9 \\
\hline 6 & 16,7 \\
\hline 6,5 & 14,9 \\
\hline 7 & 12,8 \\
\hline 7,5 & 11 \\
\hline 8 & 9,2 \\
\hline 8,5 & 7,6 \\
\hline 9 & 6 \\
\hline 9,5 & 4,6 \\
\hline 10 & 3,3 \\
\hline 10,5 & 2 \\
\hline
\end{tabular}

Tabla 1. Toma de datos $h=f(t)$

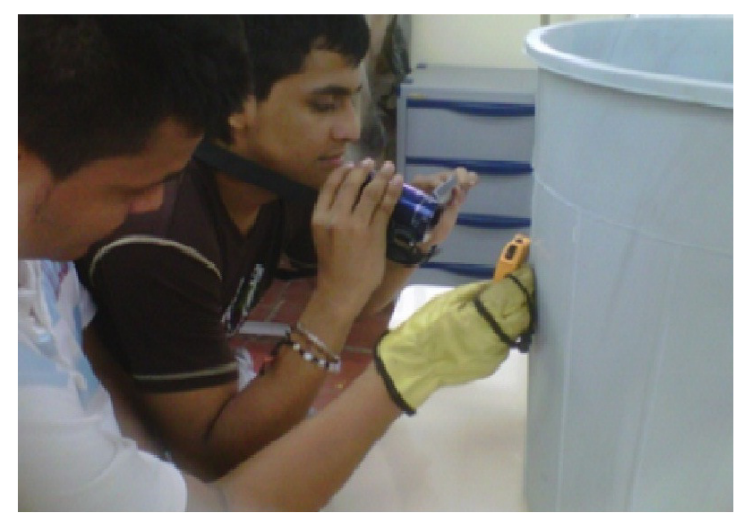

Figura 6. Proceso de filmación del Vaciado para toma de datos, por parte de estudiantes

\section{REGRESIÓN LINEAL}

Se toma inicialmente el uso del método de los mínimos cuadrados, como instrumento estadístico, y al tomar regresión lineal a los datos consignados en la tabla No. 1, por medio de la herramienta computacional Excel, se llega a los expuesto en la figura No. 7. Permitiendo llegar a una expresión del tipo $h=f(t)$, como se muestra en (5)

$h=f(t)=-4.0436 t+42.88$

con un coeficiente de determinación $R^{2}=0.9886$, el cual es muy alto, pero que puede ser mejorado.

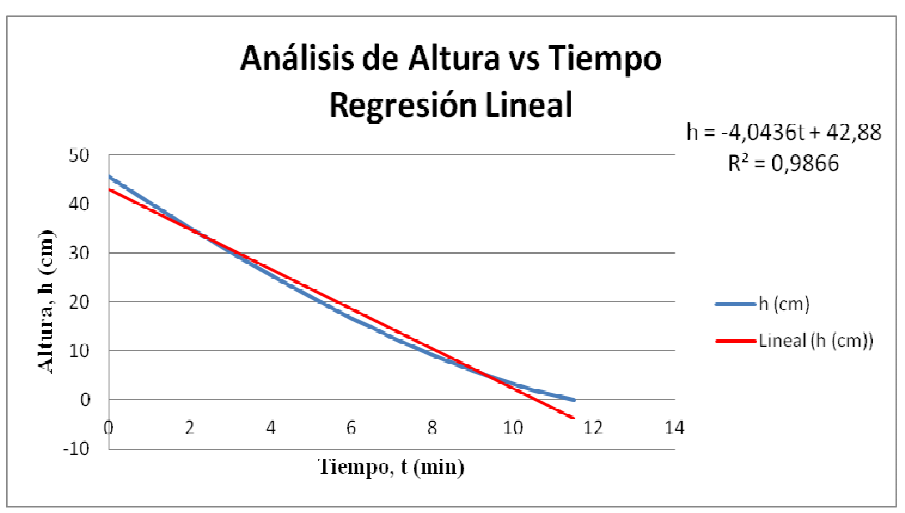

Figura 7. Análisis de Altura vs Tiempo. Regresión Lineal

\section{DIFERENCIACIÓN NUMÉRICA}

Para llevar a cabo dicha diferenciación, se utiliza el método numérico a tres pasos con diferencias centradas tal como lo propone [1], en el que se expone que la derivada puede ser encontrada a partir de una expansión por serie de Taylor, por 
medio de aproximaciones de las derivadas por diferencias divididas finitas, llegando a:

$$
f^{\prime}(t)=\frac{f(t+h)-f(t-h)}{2 h}
$$

donde $\mathrm{h}$ representa el tamaño de paso que se le ha dado a la variable independiente, en este caso el tiempo $t$. Como se pudo observar en la tabla No. 1, dicho tamaño de paso es $h=0,5$, por lo que en (6) el denominador se hace igual a 1 , permitiendo facilidad en los cálculos.

Partiendo de los datos obtenidos en el cuadro No. 1.1, se construyó la tabla No. 2, como sigue:

\begin{tabular}{|c|c|c|}
\hline $\mathbf{t}(\mathbf{m i n})$ & $\mathbf{h}(\mathbf{c m})$ & $\mathbf{d h} / \mathbf{d t}=\mathbf{\Delta h} /(\mathbf{2} \mathbf{\Delta})$ \\
\hline 0 & 45,7 & \\
\hline 0,5 & 43 & $-5,3$ \\
\hline 1 & 40,4 & $-5,2$ \\
\hline 1,5 & 37,8 & $-5,2$ \\
\hline 2 & 35,2 & -5 \\
\hline 2,5 & 32,8 & $-4,9$ \\
\hline 3 & 30,3 & $-4,9$ \\
\hline 3,5 & 27,9 & $-4,7$ \\
\hline 4 & 25,6 & $-4,6$ \\
\hline 4,5 & 23,3 & $-4,5$ \\
\hline 5 & 21,1 & $-4,4$ \\
\hline 5,5 & 18,9 & $-4,4$ \\
\hline 6 & 16,7 & -4 \\
\hline 6,5 & 14,9 & $-3,9$ \\
\hline 7 & 12,8 & $-3,9$ \\
\hline 7,5 & 11 & $-3,6$ \\
\hline 8 & 9,2 & $-3,4$ \\
\hline 8,5 & 7,6 & $-3,2$ \\
\hline 9 & 6 & -3 \\
\hline 9,5 & 4,6 & $-2,7$ \\
\hline 10 & 3,3 & $-2,6$ \\
\hline 10,5 & 2 & $-2,3$ \\
\hline 11 & 1 & -2 \\
\hline 11,5 & 0 & \\
\hline & & \\
\hline
\end{tabular}

Tabla 2. Calculo de la derivada $h=f(t)$

Teniendo en cuenta que en la tabla No. 2, la primera y la última filas, no presentan calculo de derivada, por la forma que tiene (6), se extrae la tabla No. 3 , tomando valores positivos en la columna correspondiente a $d h / d t$ con la que se procede a realizar los diferentes tipos de regresión.

Al hacer ajuste de curva por el método de mínimos cuadrados, aplicando regresión lineal, exponencial y potencial [2] a los datos obtenidos en la tabla No. 3, se obtienen los siguientes resultados, que originan las respectivas ecuaciones diferenciales, así:

\begin{tabular}{|c|c|}
\hline $\mathrm{h}(\mathrm{cm})$ & $\mathrm{dh} / \mathrm{dt}$ \\
\hline 43 & 5,3 \\
\hline 40,4 & 5,2 \\
\hline 37,8 & 5,2 \\
\hline 35,2 & 5 \\
\hline 32,8 & 4,9 \\
\hline 30,3 & 4,9 \\
\hline 27,9 & 4,7 \\
\hline 25,6 & 4,6 \\
\hline 23,3 & 4,5 \\
\hline 21,1 & 4,4 \\
\hline 18,9 & 4,4 \\
\hline 16,7 & 4 \\
\hline 14,9 & 3,9 \\
\hline 12,8 & 3,9 \\
\hline 11 & 3,6 \\
\hline 9,2 & 3,4 \\
\hline 7,6 & 3,2 \\
\hline 6 & 3 \\
\hline 4,6 & 2,7 \\
\hline 3,3 & 2,6 \\
\hline 2 & 2,3 \\
\hline 1 & 2 \\
\hline
\end{tabular}

Tabla No. 3 Calculo de la derivada para $h^{\prime}=f(t)$

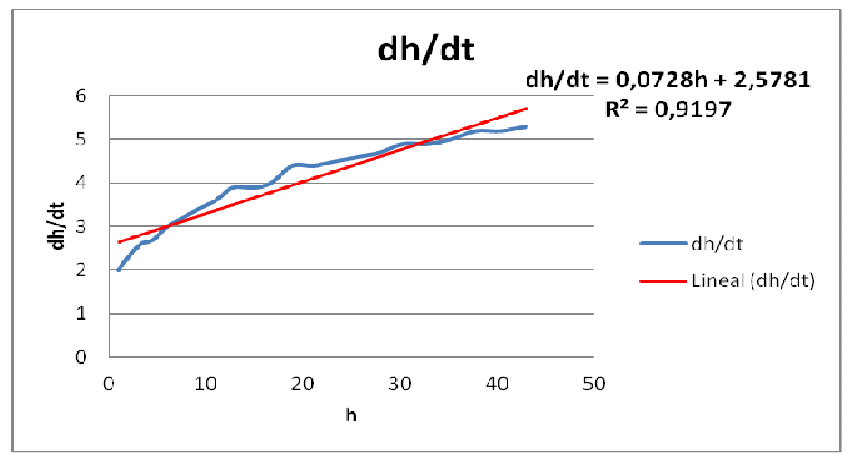

Figura 8: Regresión Lineal- Análisis derivada numérica

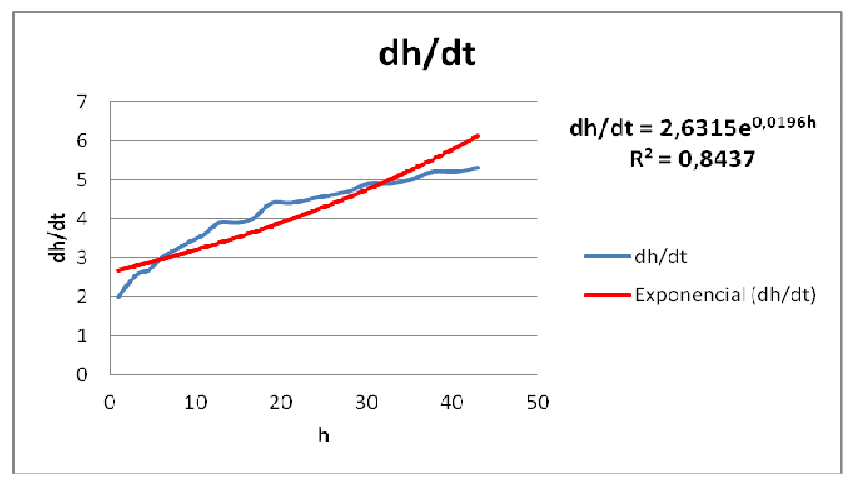

Figura 9: Regresión Exponencial- Análisis derivada numérica 


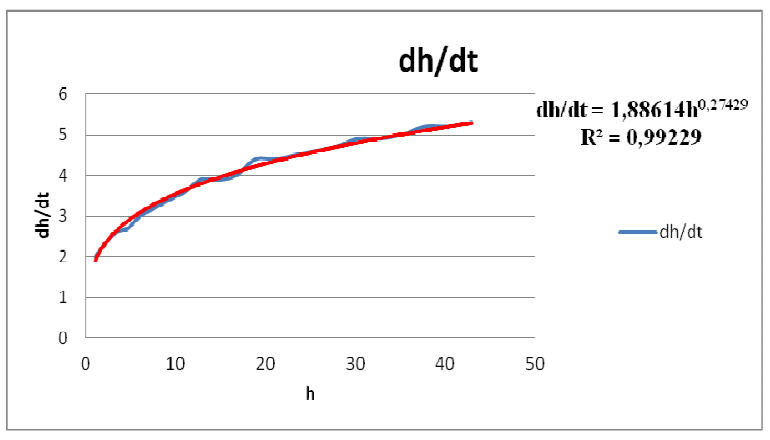

Figura 10: Regresión Potencial- Análisis derivada numérica

A continuación se da el cuadro No. 4, donde se resumen los resultados obtenidos en las figuras $(8,9$ y 10$)$.

\begin{tabular}{|c|c|c|}
\hline Regresión & Ecuación Diferencial & $R^{2}$ \\
\hline \hline Lineal & $\frac{d h}{d t}=-(0,07283 h+2,57805)$ & 0.91972 \\
\hline Exponencial & $\frac{d h}{d t}=-\left(2,63151 e^{0,01965 h}\right)$ & 0.84366 \\
\hline Potencial & $\frac{d h}{d t}=-\left(1,88614 h^{0,27429}\right)$ & 0.99229 \\
\hline
\end{tabular}

Cuadro 4. Calculo de la derivada para regresión. h'=f(t)

Se observa que el signo menos, que aparece en el cuadro No. 4, en la columna de la expresión matemática, corresponde al signo que se obvió del cuadro No. 2 , y así de esa manera se obtienen las ecuaciones diferenciales cuyas soluciones están en el cuadro No. 5, donde se adoptó como la condición inicial $\mathrm{h}(0)=45 ; 7$, tomado del cuadro No. 1.

\begin{tabular}{|c|c|c|}
\hline Regresión & Expresión Matemática $h=f(t)$ & $R^{2}$ \\
\hline \hline Lineal & $h=81,09819 e^{-0,07283 t}-35,3982$ & 0.999461 \\
\hline Exponencial & $h=-50,8906 \ln [0,05171 t+0,4073816]$ & 0.998896 \\
\hline Potencial & $h=(-1,368761 t+16,01835)^{1,37796}$ & 0.999965 \\
\hline
\end{tabular}

Cuadro 5. Diferentes modelos matemáticos para el vaciado de un tanque.

\section{ANÁLISIS DE RESULTADOS}

El análisis del cuadro No. 5 permite evidenciar que si se mejoró la expresión matemática planteada en (1.5), que era lo que se había planteado inicialmente, e implementando sus modelos obtenidos para generar los resultados expuestos en el cuadro No. 6, para cada uno de los tiempos, así como la respectiva comparación con los datos reales, tal como se presentaron inicialmente.
El mejor modelo lo representa la regresión potencial de la ecuación diferencial original, donde su coeficiente de determinación es $R^{2}=0.999965$.

\begin{tabular}{|c|c|c|c|c|}
\hline $\mathbf{t}(\mathbf{m i n})$ & $\begin{array}{c}\mathbf{h}(\mathbf{c m}) \\
\text { real }\end{array}$ & $\begin{array}{c}\text { h aproxim. } \\
\text { lineal }\end{array}$ & $\begin{array}{c}\text { h aproxim. } \\
\text { exponencial }\end{array}$ & $\begin{array}{c}\text { h aproxim. } \\
\text { potencial }\end{array}$ \\
\hline 0 & 45,7 & 45,69999 & 45,7000102 & 45,69989 \\
\hline 0,5 & 43 & 42,79992 & 42,56853 & 43,03131 \\
\hline 1 & 40,4 & 40,00356 & 39,61861 & 40,40739 \\
\hline 1,5 & 37,8 & 37,30720 & 36,83037 & 37,82941 \\
\hline 2 & 35,2 & 34,70726 & 34,18698 & 35,29875 \\
\hline 2,5 & 32,8 & 32,20029 & 31,67415 & 32,81692 \\
\hline 3 & 30,3 & 29,78297 & 29,27958 & 30,38556 \\
\hline 3,5 & 27,9 & 27,45210 & 26,99263 & 28,00645 \\
\hline 4 & 25,6 & 25,20457 & 24,80405 & 25,68154 \\
\hline 4,5 & 23,3 & 23,03742 & 22,70573 & 23,41302 \\
\hline 5 & 21,1 & 20,94777 & 20,69051 & 21,20328 \\
\hline 5,5 & 18,9 & 18,93284 & 18,75205 & 19,05501 \\
\hline 6 & 16,7 & 16,98996 & 16,88474 & 16,97127 \\
\hline 6,5 & 14,9 & 15,11656 & 15,08352 & 14,95551 \\
\hline 7 & 12,8 & 13,31016 & 13,34388 & 13,01175 \\
\hline 7,5 & 11 & 11,56835 & 11,66175 & 11,14466 \\
\hline 8 & 9,2 & 9,88883 & 10,03345 & 9,35982 \\
\hline 8,5 & 7,6 & 8,26937 & 8,45563 & 7,66400 \\
\hline 9 & 6 & 6,70782 & 6,92526 & 6,06567 \\
\hline 9,5 & 4,6 & 5,20211 & 5,43958 & 4,57575 \\
\hline 10 & 3,3 & 3,75024 & 3,99604 & 3,20916 \\
\hline 10,5 & 2 & 2,35030 & 2,59232 & 1,98775 \\
\hline 11 & 1 & 1,00041 & 1,22628 & 0,94799 \\
\hline 11,5 & 0 & $-0,30120$ & $-0,10405$ & 0,17102 \\
\hline & $\mathbf{R} 2$ & 0,999605 & 0,998924 & 0,999964 \\
\hline
\end{tabular}

Cuadro 6. Análisis de Resultados.

\section{RECOMENDACIONES}

Se recomienda realizar la practica con otro tipo de recipiente, de geometría distinta, así como con otro tipo se líquidos. Es importante analizar la opción del llenado como proceso inverso.

\section{REFERENCIAS}

[1] S. C. Chapra, R. P. Canale, Métodos numéricos para Ingenieros, Mc Graw Hill, 3 a edición, México D.F., (2000). 
[2] R. E. Walpole, R.H. Myers, S.L. Myers, Probabilidad y Estadística para ingenieros, Editorial Pearson Educación, 6 ${ }^{a}$ Edición, México D.F., p. 362. (1998).

[3] D. G. Zill, Ecuaciones diferenciales con problemas de valores en la frontera, Thomson Learning, $5^{\text {a }}$ edición, México D.F., p. 16, (2002). 